\title{
Teichmüller Curves in Genus Two: Torsion Divisors and Ratios of Sines
}

\section{Citation}

McMullen, Curtis T. 2006. Teichmüller curves in genus two: Torsion divisors and ratios of sines. Inventiones Mathematicae 165(3): 651-672. Revised 2007.

\section{Published Version}

doi:10.1007/s00222-006-0511-2

\section{Permanent link}

http://nrs.harvard.edu/urn-3:HUL.InstRepos:3446015

\section{Terms of Use}

This article was downloaded from Harvard University's DASH repository, and is made available under the terms and conditions applicable to Other Posted Material, as set forth at http:// nrs.harvard.edu/urn-3:HUL.InstRepos:dash.current.terms-of-use\#LAA

\section{Share Your Story}

The Harvard community has made this article openly available.

Please share how this access benefits you. Submit a story.

Accessibility 


\title{
Teichmüller curves in genus two: Torsion divisors and ratios of sines
}

\author{
Curtis T. McMullen
}

29 September, 2004

\section{Contents}

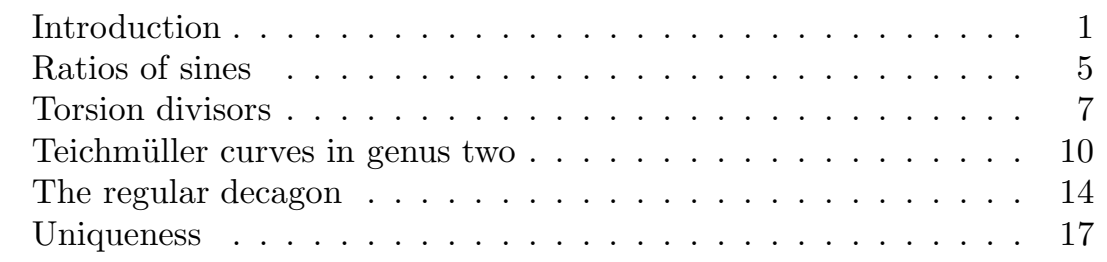

Research supported in part by the NSF and the Guggenheim Foundation. 


\section{Introduction}

Let $\mathcal{M}_{g}$ denote the moduli space of Riemann surfaces of genus $g$, and $\Omega \mathcal{M}_{g} \rightarrow$ $\mathcal{M}_{g}$ the bundle of pairs $(X, \omega)$, where $\omega \neq 0$ is a holomorphic 1-form on $X \in \mathcal{M}_{g}$.

There is a natural action of $\mathrm{GL}_{2}^{+}(\mathbb{R})$ on $\Omega \mathcal{M}_{g}$, whose orbits project to complex geodesics in $\mathcal{M}_{g}$. The projection of an orbit is almost always dense. If the stabilizer $\mathrm{SL}(X, \omega) \subset \mathrm{SL}_{2}(\mathbb{R})$ of a given form is a lattice, however, then the projection of its orbit gives a closed, algebraic Teichmüller curve

$$
f: V=\mathbb{H} / \mathrm{SL}(X, \omega) \rightarrow \mathcal{M}_{g} .
$$

A Teichmüller curve is primitive if it does not arise from a curve in $\mathcal{M}_{h}$, $h<g$, via a covering construction. Our main result completes the classification of primitive Teichmüller curves in genus two.

Theorem 1.1 The decagon form $\omega=d x / y$ on the curve $y^{2}=x\left(x^{5}-1\right)$ generates the only primitive Teichmüller curve $f: V \rightarrow \mathcal{M}_{2}$ coming from a form with simple zeros.

In contrast to this uniqueness, in genus two there are countably many primitive Teichmüller curves coming from forms with double zeros. Each such curve is uniquely determined by its discriminant and its spin invariant [Mc3].

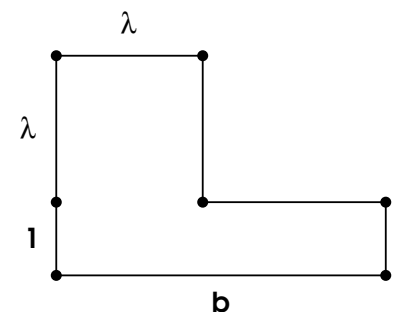

Figure 1. The billiard table $L(b, e)$, with $\lambda=\left(e+\sqrt{e^{2}+4 b}\right) / 2$.

Billiards. As a corollary of Theorem 1.1, we obtain a related classification of billiards in polygons.

Let $P \subset \mathbb{C}$ be a polygon with angles in $\pi \mathbb{Q}$. Via an unfolding construction, $P$ determines a holomorphic 1-form $(X, \omega) \in \Omega \mathcal{M}_{g}$ such that billiard trajectories in $P$ correspond to geodesics on $(X,|\omega|)$. We refer to $g$ as the genus of $P$.

For example, let $P$ denote the right triangle with smallest internal angle $\pi / 10$. Then $P$ unfolds, under repeated reflection through its long sides, to give a tiling of the regular decagon $Q$ by 20 triangles. The associated 1 -form $(X, \omega)$ is obtained from $(Q, d z)$ by identifying opposite sides; it has genus two, and agrees with the decagon form cited above.

We say $P$ and $P^{\prime}$ are equivalent if $A \cdot(X, \omega)=\left(X^{\prime}, \omega^{\prime}\right)$ for some $A \in \mathrm{GL}_{2}^{+}(\mathbb{R})$. Equivalence is a form of scissors congruence that respects the billiard dynamics. 
Veech showed that if $\operatorname{SL}(X, \omega)$ is a lattice, then the billiard flow in $P$ is dynamically optimal: every trajectory is either periodic or uniformly distributed, and the number of types of periodic trajectories of length $\leq L$ is asymptotic to $c(P) \cdot L^{2} ;$ see $[\mathrm{V}]$.

Theorem 1.2 Let $P \subset \mathbb{C}$ be a polygon of genus two. Then the billiard flow in $P$ is dynamically optimal if and only if $P$ is equivalent to:

- A polygon tiled by congruent 30-60-90 triangles or isoceles right triangles;

- The L-shaped polygon $L(b, e)$, for some $b, e \in \mathbb{Z}$ (Figure 1); or

- The 18-72-90 triangle that tiles the regular decagon.

Otherwise, there is a billiard trajectory in $P$ that is neither periodic nor dense.

Proof. By [Mc4, Thm. 7.1], either $\operatorname{SL}(X, \omega)$ is a lattice, or $P$ has a trajectory that is neither periodic nor dense.

Assume we are in the former case. Then $P$ is dynamically optimal, and $(X, \omega)$ generates a Teichmüller curve $f: V \rightarrow \mathcal{M}_{2}$.

If $\omega$ has a double zero, then $P$ is equivalent to an $L$-shaped polygon $L(b, e)$ [Mc3, Cor. 1.3], and all such polygons are optimal

If $\omega$ has a pair of simple zeros and the Teichmüller curve it generates is primitive, then $P$ is equivalent to the 18-72-90 triangle by Theorem 1.1.

Otherwise, $\omega$ is the pullback of a form of genus one via a covering $\pi: X \rightarrow$ $\mathbb{C} / \Lambda$ branched over just one point. We can assume $\Lambda$ is the square or hexagonal lattice, and thus $P$ is equivalent to a polygon tiled as above. (Examples of this type include rectangles with rational barriers [EMS].) Conversely $\operatorname{SL}(X, \omega)$ is commensurable to $\mathrm{SL}_{2}(\mathbb{Z})$ whenever $P$ can be tiled in this way, and thus $P$ is optimal.

Infinitely generated groups. Theorem 1.1 also yields a large family of examples where $\operatorname{SL}(X, \omega)$ is infinitely generated.

Theorem 1.3 Let $(X, \omega)$ be a form of genus two with simple zeros, such that the trace field of $\operatorname{SL}(X, \omega)$ is irrational. Then either:

- $(X, \omega)$ is equivalent to the decagon form, or

- $\operatorname{SL}(X, \omega)$ is an infinitely generated group.

This follows from $[\mathrm{Mc} 2, \S 10]$.

Torsion divisors. The proof of our main result relies on:

Theorem 1.4 (Möller) Suppose $\operatorname{SL}(X, \omega)$ is a lattice with trace field $K$, and $\operatorname{deg}(K / \mathbb{Q})$ agrees with the genus of $X$. Then the divisor $[P-Q]$ has finite order in $\operatorname{Jac}(X)$, whenever $P$ and $Q$ are two zeros of $\omega$. 
See [Mo, Cor 3.4]. The proof also uses an elementary fact about cyclotomic fields $(\S 2)$ :

Theorem 1.5 There are only 15 pairs of rational numbers $0<\alpha<\beta<1 / 2$ such that $\sin (\pi \alpha) / \sin (\pi \beta)$ is a quadratic irrational.

To see how these results are related to Theorem 1.1, let $f: V \rightarrow \mathcal{M}_{2}$ be a primitive Teichmüller curve generated by a form $(X, \omega) \in \Omega \mathcal{M}_{2}$ with simple zeros. Normalizing by the action of $\mathrm{GL}_{2}^{+}(\mathbb{R})$, we can assume

$$
(X, \omega)=\left(E_{1}, \omega_{1}\right) \underset{I}{\#}\left(E_{2}, \omega_{2}\right)
$$

is the connected sum of two forms of genus one, with period lattices given by

$$
\begin{aligned}
& \operatorname{Per}\left(\omega_{1}\right)=\mathbb{Z}(\lambda, 0) \oplus \mathbb{Z}(0, \lambda), \quad \lambda=\left(e+\sqrt{e^{2}+4 b c}\right) / 2, \quad \text { and } \\
& \operatorname{Per}\left(\omega_{2}\right)=\mathbb{Z}(b, 0) \oplus \mathbb{Z}(a, c)
\end{aligned}
$$

for suitable integers $(a, b, c, e)$ (see $\S 4)$. We can then use the diagonal matrices $A_{s}=\left(\begin{array}{ll}1 & 0 \\ 0 & s\end{array}\right)$ to push $(X, \omega)$ to the boundary of moduli space, and obtain a limiting stable Abelian differential $(Y, d x / y)$. The limit $Y$ is a stable curve of genus zero with two nodes, that can be expressed in the form

$$
y^{2}=\left(1-x^{2}\right)(x-A)^{2}(x-B)^{2}
$$

with $-1<A<B<1$. (The nodal curve that arises from the decagon form, determined in $\S 5$, is shown in Figure 2.)

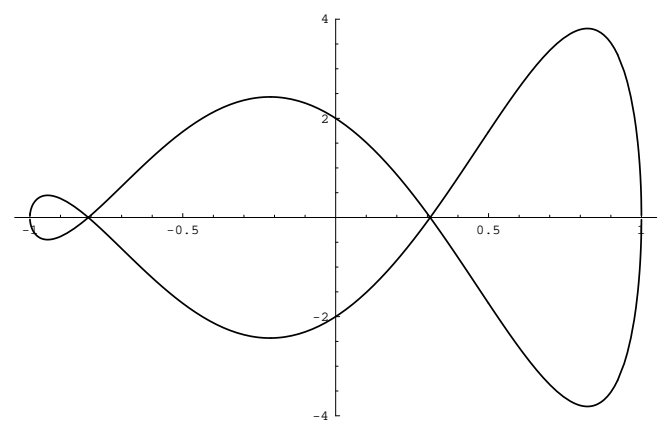

Figure 2. A nodal curve in the boundary of $\mathcal{M}_{2}$.

Writing $(A, B)=(-\cos \pi \alpha, \cos \pi \beta)$ with $0<\alpha, \beta<1$, we find

$$
\frac{\sin (\pi \alpha)}{\sin (\pi \beta)}=\frac{\lambda}{b}
$$

by a residue calculation $(\S 4)$. On the other hand, by Theorem 1.4 the divisor $D_{\infty}=P-Q$ is torsion in $\operatorname{Jac}(Y)$, where $(d x / y)=P+Q$ consists of the two 
points in $Y$ lying over $x=\infty$. But it is straightforward to show if $D_{\infty}$ is torsion in $\operatorname{Jac}(Y)$, then $\alpha$ and $\beta$ are rational ( $(2)$. Thus (1.1) must agree with one of the 15 quadratic irrational sine ratios occurring in Theorem 2.1.

This fact radically constrains the possibilities for $(X, \omega)$, leading to 22 cases listed in $\S 6$. Only the decagon form (which comes from $\sin (\pi / 5) / \sin (2 \pi / 5)=$ $(-1+\sqrt{5}) / 2)$ passes a further rationality test, completing the proof.

Curves on Hilbert modular surfaces. We conclude by formulating a purely algebraic corollary of Theorem 1.1.

Let $D>0$ be the discriminant of an order $\mathcal{O}_{D}$ in a real quadratic field. For each $n>0$, let $\Omega E_{D}[n] \subset \mathcal{M}_{2}$ denote the set of forms $(X, \omega)$ such that

- $\operatorname{Jac}(X)$ admits real multiplication by $\mathcal{O}_{D}$, with $\omega$ as an eigenform, and

- The divisor $[P-Q]$ has order $n$ in $\operatorname{Jac}(X)$, where $P$ and $Q$ are the zeros of $\omega$.

We denote the projection of $\Omega E_{D}[n]$ to $\mathcal{M}_{2}$ by $W_{D}[n]$. The locus $W_{D}[1]$ is called the Weierstrass curve in [Mc3].

Theorem 1.6 The variety $W_{D}[1]$ is 1-dimensional for all $D$, as is the locus $W_{5}[5]$. Otherwise $W_{D}[n]$ is zero-dimensional.

This result follows from:

Theorem 1.7 The primitive Teichmüller curves generated by forms of genus two coincide with the 1-dimensional irreducible components of $\bigcup W_{D}[n]$.

Proof. Let $\Omega R_{D} \subset \Omega \mathcal{M}_{2}$ denote the set of eigenforms $(X, \omega)$ for real multiplication by $\mathcal{O}_{D}$ such that the relative periods of $\omega$ lie in the rational span of its absolute periods, i.e.

$$
\int_{P}^{Q} \omega \in \operatorname{Per}(\omega) \otimes \mathbb{Q}
$$

where $P$ and $Q$ are the zeros of $\omega$. The locus $\Omega R_{D}$ is a countable union of $\mathrm{GL}_{2}^{+}(\mathbb{R})$-orbits, so its projection $R_{D} \subset \mathcal{M}_{2}$ is a countable union of complex geodesics.

By the Abel-Jacobi Theorem, if $n(P-Q)$ is a principal divisor then $n \int_{P}^{Q} \xi$ is a period of $\xi$ for every $\xi \in \Omega(X)$. Thus $W_{D}[n] \subset R_{D}$, so $\operatorname{dim} W_{D}[n] \leq 1$.

Now let $f: V \rightarrow \mathcal{M}_{2}$ be a primitive Teichmüller curve. Then $f(V)$ is contained in the locus of real multiplication by [Mc1], and $f(V) \subset W_{D}[n]$ by Theorem 1.4. Conversely, if $V \subset W_{D}[n]$ is an irreducible curve, then $V$ is also an algebraic component of $R$. Hence $V$ is a Teichmüller curve, which is primitive because $D$ is not a square.

Proof of Theorem 1.6. As we will see in $\S 5$, the curve generated by the decagon form lies in $W_{5}[5]$. Moreover, the forms in $\Omega W_{D}[n]$ have simple zeros iff $n>1$. Thus the theorem is immediate from [Mc3] when $n=1$, and from Theorem 1.1 when $n>1$. 
It would be interesting to have a similar algebraic description of the primitive Teichmüller curves in higher genus.

Notes and references. Theorem 1.1 was conjectured in [Mc3], and established for all Teichmüller curves with discriminant $D \leq 400$ in [Mc4]. For additional results related to Teichmüller curves in genus two, see [V], [GJ], [EMS], [HS], $[\mathrm{HL}]$, and $[\mathrm{Ca}]$. I would like to thank J. Harris and M. Möller for useful discussions.

\section{Ratios of sines}

In this section we will show:

Theorem 2.1 For each $d>0$, there are only finitely many pairs of rational numbers $0<\alpha<\beta \leq 1 / 2$ such that

$$
\sin (\pi \alpha) / \sin (\pi \beta)
$$

is an algebraic number of degree d over $\mathbb{Q}$.

Lemma 2.2 Let $f_{t}(x)=\sin (t x) /(t \sin (x))$ with $t>1$. If $x_{1}, t x_{1}$ and $x_{2}$ all lie in the interval $[0, \pi / 2]$, and $f_{t}\left(x_{1}\right)=f_{t}\left(x_{2}\right)$, then $x_{1}=x_{2}$.

Proof. Since $2 / \pi \leq \sin (x) / x \leq 1$ for all $x \in[0, \pi / 2]$, we have $f_{t}\left(x_{1}\right) \geq 2 / \pi \geq$ $1 / 2$, which implies

$$
(2 / \pi) x_{2} \leq \sin \left(x_{2}\right) \leq \frac{\sin \left(x_{2}\right)}{\left|\sin \left(t x_{2}\right)\right|}=\frac{1}{\left|t f_{t}\left(x_{2}\right)\right|}=\frac{1}{t f_{t}\left(x_{1}\right)} \leq 2 / t .
$$

Thus $x_{1}$ and $x_{2}$ both belong to the interval $[0, \pi / t]$. But it is easy to see that $f_{t}(x)$ is strictly decreasing on this interval (with $f_{t}(0)=1$ and $f_{t}(\pi / t)=0$ ), so the condition $f_{t}\left(x_{1}\right)=f_{t}\left(x_{2}\right)$ implies $x_{1}=x_{2}$.

Proof of Theorem 2.1. Fix a degree $d>0$, and let $A=\pi(a / p)$ and $B=$ $\pi(b / q)$ be rational multiples of $\pi$ in lowest terms, such that $0<A<B<\pi / 2$ and $\sin (A) / \sin (B)$ has degree $d$ over $\mathbb{Q}$. To show there are only finitely many possibilities for the pair $(a / p, b / q)$, we will show that $n=\operatorname{lcm}(p, q)$ is bounded by a constant $N_{d}$ depending only on $d$.

Consider the field extension $L / K$, where $K=\mathbb{Q}(\sin (A))$ and $L=K(\sin (B))$. Then $\operatorname{deg}(K / \mathbb{Q})$ and $\operatorname{deg}(L / \mathbb{Q})$ are comparable to $\phi(p)$ and $\phi(n)$ respectively, where $\phi$ is Euler's phi-function. We also have $\operatorname{deg}(L / K) \leq d$, so $\phi(n) / \phi(p)=$ $O(d)$, which implies $(n / p) \leq C_{d}$ for some constant $C_{d}$.

Next, recall that the Galois conjugates of $\sin (A) / \sin (B)$ are given by

$$
\mu(k)=\sin (k A) / \sin (k B),
$$

where $k$ ranges in

$$
(\mathbb{Z} / 2 n)^{*} \cong \operatorname{Gal}\left(\mathbb{Q}\left(e^{\pi i / n}\right) / \mathbb{Q}\right)
$$




\begin{tabular}{|c|c|c|c|c|}
\hline$\alpha$ & $\beta$ & $\sin (\pi \alpha) / \sin (\pi \beta)$ & Trace & Norm \\
\hline \hline $1 / 12$ & $5 / 12$ & $2-\sqrt{3}$ & 4 & 1 \\
\hline $1 / 10$ & $1 / 2$ & $(-1+\sqrt{5}) / 4$ & $-1 / 2$ & $-1 / 4$ \\
\hline $1 / 12$ & $1 / 4$ & $(-1+\sqrt{3}) / 2$ & -1 & $-1 / 2$ \\
\hline $1 / 10$ & $3 / 10$ & $(3-\sqrt{5}) / 2$ & 3 & 1 \\
\hline $1 / 8$ & $3 / 8$ & $-1+\sqrt{2}$ & -2 & -1 \\
\hline $1 / 6$ & $1 / 3$ & $\sqrt{3} / 3$ & 0 & $-1 / 3$ \\
\hline $1 / 6$ & $3 / 10$ & $(-1+\sqrt{5}) / 2$ & -1 & -1 \\
\hline $1 / 10$ & $1 / 6$ & $(-1+\sqrt{5}) / 2$ & -1 & -1 \\
\hline $1 / 5$ & $2 / 5$ & $(-1+\sqrt{5}) / 2$ & -1 & -1 \\
\hline $1 / 6$ & $1 / 4$ & $\sqrt{2} / 2$ & 0 & $-1 / 2$ \\
\hline $1 / 4$ & $1 / 2$ & $\sqrt{2} / 2$ & 0 & $-1 / 2$ \\
\hline $1 / 4$ & $5 / 12$ & $-1+\sqrt{3}$ & -2 & -2 \\
\hline $3 / 10$ & $1 / 2$ & $(1+\sqrt{5}) / 4$ & $1 / 2$ & $-1 / 4$ \\
\hline $1 / 4$ & $1 / 3$ & $\sqrt{6} / 3$ & 0 & $-2 / 3$ \\
\hline $1 / 3$ & $1 / 2$ & $\sqrt{3} / 2$ & 0 & $-3 / 4$ \\
\hline
\end{tabular}

Table 3. The fifteen quadratic irrational sine ratios. 
Thus by replacing $\mu(1)$ with $\mu(k)$ with $k a=1 \bmod p$, we can assume $a=1$.

Let $t$ be the least integer greater than 1 such that $\operatorname{gcd}(t, 2 n)=1$ and $\mu(t)=$ $\mu(1)$. We then have

$$
\frac{\sin (t A)}{\sin (A)}=\frac{\sin (t B)}{\sin (B)} .
$$

We also have the bound $t \leq(5 \log (2 n))^{d}$, since there is an integer $1<k<$ $5 \log (2 n)$ relatively prime to $n$ [Mc3, Thm. 9.1], and $\mu\left(k^{i}\right)=\mu(1)$ for some $i \leq d$. This shows

$$
0<t A=\pi t a / p \leq \pi C_{d}(5 \log (2 n))^{d} / n,
$$

and therefore $t A<\pi / 2$ whenever $n$ exceeds a suitable constant $N_{d}$ depending only on $d$.

But if $t A<\pi / 2$, then we can apply the preceding Lemma to (2.1) to conclude that $A=B$, a contradiction. Consequently $n \leq N_{d}$, and therefore the number of sine ratios of degree $d$ over $\mathbb{Q}$ is finite.

Quadratic sine ratios. The only rational sine ratio occurring as above is $\sin (\pi / 6) / \sin (\pi / 2)=1 / 2$. Similarly, a straightforward computation using the bounds of the preceding proof establishes:

Theorem 2.3 The ratio $\sin (\pi \alpha) / \sin (\pi \beta)$ is a quadratic irrational for exactly 15 pairs of rational numbers $0<\alpha<\beta \leq 1 / 2$.

These pairs are listed in Table 3.

For related results, see [Man].

\section{Torsion divisors}

In this section we review the classical relationship between Pell's equation over $\mathbb{C}[x]$ and torsion divisors on hyperelliptic curves.

Hyperelliptic curves. Let $C_{0}$ be the hyperelliptic curve defined by the equation

$$
y^{2}=p(x),
$$

where $p(x) \in \mathbb{C}[x]$ has degree $2 g-2$ and $g \geq 0$. The curve $C_{0} \subset \mathbb{C}^{2}$ extends to a unique complete curve $C$ of arithmetic genus $g$, with two points $P, Q \in C$ lying over $x=\infty$. The completion is smooth if all the zeros of $p(x)$ are simple; otherwise, it has singularities lying over the multiple zeros of $p$.

Note that the hyperelliptic involution $(x, y) \mapsto(x,-y)$ interchanges $P$ and $Q$. We refer to $D_{\infty}=P-Q \in \operatorname{Div}(C)$ as the divisor at infinity; it is well-defined up to sign.

The divisor $n D_{\infty}$ is principal if the following equivalent conditions hold:

1. There is a regular function $f: C_{0} \rightarrow \mathbb{C}^{*}$ with a pole of order $n$ at $P$, and a zero of order $n$ at $Q$. 
2. The class $n[D] \in \operatorname{Pic}(C)$ is trivial.

3. The curve $C$ is irreducible, and $h^{0}\left(C, \mathcal{O}_{C}(n D)\right)>0$.

In case (1) we write $n D_{\infty}=(f)$. The divisor $D_{\infty}$ is torsion if $n D_{\infty}$ is principal for some $n>0$.

Theorem 3.1 The divisor $n D, n>0$ is principal if and only if there are polynomials $a, b \in \mathbb{C}[x]$, with $\operatorname{deg}(a)=n$, satisfying Pell's equation

$$
a(x)^{2}-p(x) b(x)^{2}=1 .
$$

Proof. Assume $a, b \in \mathbb{C}[x]$ satisfy (3.1). Then the polynomial $f(x, y)=a(x)+$ $y b(x) \in \mathbb{C}[x, y] /\left(y^{2}-p(x)\right)$ satisfies

$$
f(x, y) \cdot f(x,-y)=(a+y b)(a-y b)=1,
$$

so it defines a regular map $f: C_{0} \rightarrow \mathbb{C}^{*}$. Since $a(x)$ and $y b(x)$ both have poles of order $n$ at $P$ and $Q, f(x, y)$ has a pole of order $n$ at one of these points, and hence a zero of order $n$ at the other; therefore $n D$ is a principal divisor.

Conversely, suppose $n D$ is the divisor of a regular map $f: C \rightarrow \mathbb{P}^{1}$. Then the product $f(x, y) f(x,-y)=c$ is a nonzero constant, since it has no zeros or poles. Replacing $f$ with $f / \sqrt{c}$, we can assume $c=1$. Then the rational functions

$$
a(x, y)=\frac{f(x, y)+f(x,-y)}{2}, \quad b(x, y)=\frac{f(x, y)-f(x,-y)}{2 y}
$$

are invariant under the hyperelliptic involution, and have no poles on $C_{0}$; so they are actually polynomials in $x$, satisfying (3.1).

Remark. The solution to $(f)=n D_{\infty}$ is a unit in the coordinate ring

$$
\mathcal{O}\left(C_{0}\right)=\mathbb{C}[x, y] /\left(y^{2}-p(x)\right),
$$

and $a^{2}-p b^{2}$ is its norm over $\mathbb{C}[x]$.

Genus zero. Recall (e.g. from [Ri]) that the Chebyshev polynomials $T_{n}(x)$ and $U_{n}(x)$ of the first and second kinds are characterized by:

$$
T_{n}(\cos \theta)=\cos (n \theta), \quad U_{n-1}(\cos \theta)=\sin (n \theta) / \sin (\theta) .
$$

Theorem 3.2 Let $C$ be the conic $y^{2}=x^{2}-1$. Then $n D_{\infty}$ is principal for every $n>0$, and the corresponding solutions to Pell's equation are given by

$$
T_{n}(x)^{2}-\left(x^{2}-1\right) U_{n-1}(x)^{2}=1 .
$$


Proof. Under the isomorphism $\mathbb{P}^{1} \cong C$ given by

$$
t \mapsto(x(t), y(t))=\left(\frac{t+t^{-1}}{2}, \frac{t-t^{-1}}{2}\right),
$$

the points $t=0$ and $t=\infty$ lie over $x=\infty$; thus $f(t)=t^{n}$ satisfies $(f)=n D_{\infty}$. Setting $t=e^{i \theta}$, we have $(x, y)=(\cos \theta, i \sin \theta)$, and thus equation (3.2) implies that $(a, b)=\left( \pm T_{n}, \pm U_{n-1}\right)$. Indeed, Pell's equation amounts simply to the identity

$$
T_{n}(x)^{2}-\left(x^{2}-1\right) U_{n-1}(x)^{2}=\cos ^{2}(n \theta)+\sin ^{2}(n \theta)=1 .
$$

Corollary 3.3 The divisor $D_{\infty}$ is torsion on the curve

$$
y^{2}=\left(x^{2}-1\right) q(x)^{2}
$$

if and only if $q(x)$ has simple zeros, all contained in $(-1,1) \cap \cos \pi \mathbb{Q}$.

Proof. By the preceding theorem, we can solve Pell's equation

$$
a(x)^{2}-\left(x^{2}-1\right) q(x)^{2} b(x)^{2}=1
$$

if and only if $q(x)$ divides $U_{n-1}(x)$ for some $n$. Since the zeros of $U_{n-1}(x)$ coincide with the set $(-1,1) \cap \cos (\pi / n) \mathbb{Z}$, and each has multiplicity one, the result follows.

Limits. We conclude by noting that torsion divisors behave well under limits, so long as the limiting curve is irreducible.

Theorem 3.4 Let $C_{k}$ and $C$ be irreducible hyperelliptic curves of arithmetic genus $g$, defined by a convergent sequence of polynomials

$$
y^{2}-p_{k}(x) \rightarrow y^{2}-p(x) \in \mathbb{C}[x, y] .
$$

Suppose the divisor at infinity is torsion of order $\leq n$ for each $C_{k}$. Then the same is true for $C$.

Proof. By assumption there exist polynomial solutions to Pell's equation $a_{k}(x)^{2}-p_{k}(x) b_{k}(x)^{2}=1$ of uniformly bounded degree. If the coefficients of $\left(a_{k}, b_{k}\right)$ are bounded, a convergent subsequence yields a solution to the equation $a(x)^{2}-p(x) b(x)^{2}=1$, so the divisor at infinity is torsion for $C$. Otherwise, a limit of suitable rescalings of the polynomials $\left(a_{k}, b_{k}\right)$ yields a nontrivial solution to the equation $a(x)^{2}-p(x) b(x)^{2}=0$; this implies $p(x)$ is a square, contrary to irreducibility of $C$.

Remark. The sequence $y^{2}=x^{2}-1 / k^{2} \rightarrow y^{2}-x^{2}$ gives a simple example showing Theorem 3.4 fails without irreducibility.

Notes and references. The theory of torsion divisors on hyperelliptic curves was studied by Abel in [Ab]; for more recent developments, see [HMP] [BC], $[\mathrm{BGL}]$, and $[\mathrm{Yu}]$. 


\section{Teichmüller curves in genus two}

In this section we recall the theory of Teichmüller curves in genus two, and the prototypical forms that generate them. We then establish:

Theorem 4.1 If the prototypical form $(X, \omega)$ of type $(a, b, c, e)$ and width $0<$ $t<1$ generates a primitive Teichmüller curve

$$
f: V \rightarrow \mathcal{M}_{2}
$$

then there are rational numbers $0<\alpha<\beta<1 / 2$ satisfying

$$
\frac{\sin (\pi \alpha)}{\sin (\pi \beta)}=\frac{\lambda}{b} \quad \text { and } \quad t=\beta+\alpha \frac{b}{\lambda}
$$

where $\lambda=\left(e+\sqrt{e^{2}+4 b c}\right) / 2$.

In the course of the proof, we will obtain an explicit formula for certain nodal curves $Y \in \partial \mathcal{M}_{2}$ that arise from the cusps of $V$.

Teichmüller curves. Let $\mathcal{M}_{g}$ denote the moduli space of Riemann surfaces of genus $g$, and let

$$
\Omega \mathcal{M}_{g} \rightarrow \mathcal{M}_{g}
$$

denote the bundle of pairs $(X, \omega)$ such that $\omega$ is a nonzero holomorphic 1-form on $X \in \mathcal{M}_{g}$. The space $\Omega \mathcal{M}_{g}$ carries a natural action of $\mathrm{GL}_{2}^{+}(\mathbb{R})$, whose orbits project to complex geodesics in $\mathcal{M}_{g}$ (see e.g. [Mc1]). The stabilizer of a given form will be denoted by $\mathrm{SL}(X, \omega)$; it is a discrete subgroup of $\mathrm{SL}_{2}(\mathbb{R})$.

If $\mathrm{SL}(X, \omega)$ is a lattice in $\mathrm{SL}_{2}(\mathbb{R})$, then the projection of its orbit gives a holomorphic Teichmüller curve

$$
f: V=\mathbb{H} / \mathrm{SL}(X, \omega) \rightarrow \mathcal{M}_{g} .
$$

The image of $f(V) \subset \mathcal{M}_{g}$ is an algebraic curve, isometrically immersed for the Teichmüller metric. We say $f: V \rightarrow \mathcal{M}_{g}$ is primitive if the form $(X, \omega)$ is not the pullback of a holomorphic form on a curve of lower genus.

Stable curves. Let $\overline{\mathcal{M}_{g}}$ denote the compactification of moduli space by stable curves. By passing to the normalization

$$
\pi: \widetilde{Y} \rightarrow Y,
$$

any stable curve $Y \in \overline{\mathcal{M}_{g}}$ can be regarded as a smooth curve $\tilde{Y}$ with certain pairs of points $\left(y_{i}, y_{i}^{\prime}\right)$ identified to form nodes.

Let $\Omega(Y)$ denote the space of holomorphic 1-forms on $\tilde{Y}-\bigcup\left\{y_{i}, y_{i}^{\prime}\right\}$ with at worst simple poles, of opposite residues, at each pair of points $\left(y_{i}, y_{i}^{\prime}\right)$. Then $\Omega \mathcal{M}_{g} \rightarrow \mathcal{M}_{g}$ extends to the bundle

$$
\Omega \overline{\mathcal{M}_{g}} \rightarrow \overline{\mathcal{M}_{g}}
$$


of stable Abelian differentials, consisting of pairs $(Y, \xi)$ with $\xi \in \Omega(Y)$. This bundle can be regarded as the relative dualizing sheaf for the universal curve $\overline{\mathcal{C}_{g}} \rightarrow \overline{\mathcal{M}_{g}}[\mathrm{HM}$, Ch. 3].

Prototypical forms. We now specialize to the case $g=2$. Following [Mc4, $\S 3]$, we say a set of integers $p=(a, b, c, e)$ is a splitting prototype of discriminant $D>0$ if:

$$
\begin{array}{lll}
D=e^{2}+4 b c, & 0 \leq a<\operatorname{gcd}(b, c), & c+e<b, \\
0<b, & 0<c, \text { and } & \operatorname{gcd}(a, b, c, e)=1 .
\end{array}
$$

Let $\lambda=(e+\sqrt{D}) / 2$ and let $I=[0, t \lambda]$, where $t \in(0,1]$. Let $\left(E_{i}, \omega_{i}\right)=$ $\left(\mathbb{C} / \Lambda_{i}, d z\right), i=1,2$ be the forms of genus one with period lattices $\Lambda_{1}=\mathbb{Z}(\lambda, 0) \oplus$ $\mathbb{Z}(0, \lambda)$ and $\Lambda_{2}=\mathbb{Z}(b, 0) \oplus \mathbb{Z}(a, c)$.

The prototypical form of type $p$ and width $t$ is given by the connected sum

$$
(X, \omega)=\left(E_{1}, \omega_{1}\right) \underset{I}{\#}\left(E_{2}, \omega_{2}\right) .
$$

The connected sum is obtained by slitting each torus open along the projection of the $\operatorname{arc} I$, and then gluing corresponding edges. The form $(X, \omega)$ can also be expressed geometrically as the quotient $(P, d z) / \sim$ of the polygon $P \subset \mathbb{C}$ shown in Figure 4.

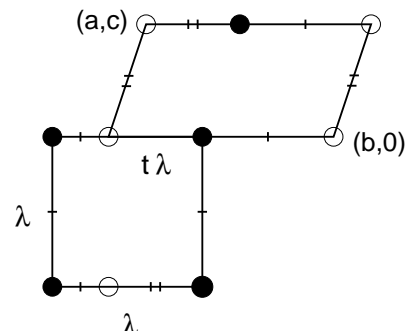

Figure 4. The prototypical form of type $(a, b, c, e)$ and width $t$.

By [Mc4, $§ 1$ and Cor. 3.7] we have:

Theorem 4.2 Every Teichmüller curve generated by a form in $\Omega \mathcal{M}_{2}$ is also generated by a prototypical form. The resulting curve is primitive if and only if the discriminant $D=e^{2}+4 b c$ is not a square.

Stable sums. The operation of connected sum extends continuously way to the case where the tori $E_{i} \cong \mathbb{C} / \Lambda_{i}$ degenerate to cylinders $C_{i} \cong \mathbb{C} / \mathbb{Z} a_{i} \cong \mathbb{C}^{*}$. In this case the connected sum gives a stable curve in the stratum $\mathcal{M}_{0,4} \subset \partial \mathcal{M}_{2}$, with the ends $\left(y_{i}, y_{i}^{\prime}\right)$ of each cylinder $C_{i}$ identified to form nodes. 
Theorem 4.3 Let $I=[0, v]$, and assume $0<v<r<s$. Then the connected sum

$$
(Y, \omega)=(\mathbb{C} / \mathbb{Z} r, d z) \underset{I}{\#}(\mathbb{C} / \mathbb{Z} s, d z)
$$

is proportional to the stable Abelian differential $d x / y$ on a nodal curve of the form

$$
y^{2}=\left(x^{2}-1\right)(x+\cos (\pi \alpha))^{2}(x-\cos (\pi \beta))^{2},
$$

where $\alpha \in(0,1 / 2), \beta \in(0,1), \sin (\pi \alpha) / \sin (\pi \beta)=r / s$ and $v=\alpha s+\beta r$.

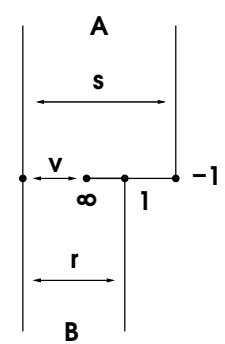

Figure 5. Connected sum of cylinders.

Proof. Start by expressing $Y$ as a degree two branched covering $\pi: Y \rightarrow \mathbb{P}^{1}$ of the projective line, with hyperelliptic involution $\eta$. Then the form $\left(Y, \omega^{2}\right) / \eta$ gives a meromorphic quadratic differential $\left(\mathbb{P}^{1}, q\right)$ on the quotient $\mathbb{P}^{1}$. Since the periods $r, s$ and $v$ of $\omega$ are real, $\left(\mathbb{P}^{1}, q\right)$ can be constructed from a rectangular polygon $\left(P,(d z / 2)^{2}\right)$ in $\mathbb{C}$. More precisely, if we define $P$ by gluing the infinite rectangles $[0, s] \times[0, \infty)$ and $[0, r] \times[0,-\infty)$ together along the segment $[0, v]$, then the double of $\left(P, d z^{2} / 4\right)$ along its edges can be identified with $\left(\mathbb{P}^{1}, q\right)$.

We can normalize so that the interior of $P$ corresponds to the upper half plane $\mathbb{H} \subset \mathbb{P}^{1}$, its boundary to the extended real axis, and its vertices to the ordered set $(\infty,-1, A, B, 1) \subset \mathbb{R}$. The image of $I$ on $\mathbb{P}^{1}$ is then a loop based at $\infty$, crossing the real axis between $A$ and $B$.

Since $-1<A<B<1$, there are unique constants $0<\alpha, \beta<1$ such that $(-\cos \pi \alpha, \cos \pi \beta)=(A, B)$. Since $\pi: Y \rightarrow \mathbb{P}^{1}$ is branched over $x= \pm 1$ and has nodes over $x=A, B, Y$ is isomorphic to the hyperelliptic curve (4.3), with $\pi(x, y)=x$. Therefore $\omega=C d x / y$, for some $C \neq 0$, since these two forms have the same zeros and poles. Consequently we have

$$
q=\omega^{2}=\frac{C^{2} d x^{2}}{\left(x^{2}-1\right)(x-A)^{2}(x-B)^{2}},
$$

which implies

$$
\frac{\operatorname{Res}_{B}(q)}{\operatorname{Res}_{A}(q)}=\left(\frac{r}{s}\right)^{2}=\frac{1-A^{2}}{1-B^{2}}=\left(\frac{\sin (\pi \alpha)}{\sin (\pi \beta)}\right)^{2} .
$$


Thus $\sin (\pi \alpha) / \sin (\pi \beta)=r / s$, because both sides are positive.

Using the fact that $\operatorname{Res}_{A}(q)=s^{2} /(2 \pi i)^{2}$ and $\operatorname{Res}_{B}(q)=r^{2} /(2 \pi i)^{2}$, it is then straightforward to compute

$$
v=\int_{I} \omega=\int_{-i \infty}^{+i \infty} \sqrt{q}=\alpha s+\beta r
$$

where the integral is taken along a path that crosses $\mathbb{R}$ between $A$ and $B$. The choice of square-root is dictated by the condition $v>0$.

Finally note that the inequalities $r<s$ and $A<B$ imply

$$
0 \leq \sin (\pi \alpha)<\sin (\pi \beta) \text { and }-\cos (\pi \alpha)<\cos (\pi \beta) ;
$$

since $\sin ^{2}(\theta)+\cos ^{2}(\theta)=1$, we must have $-\cos (\pi \alpha)<0$ and therefore $\alpha<1 / 2$.

Theorem 4.4 Let $(X, \omega)$ be the prototypical form of type $(a, b, c, e)$ and width $t$, and let $A_{s}=\left(\begin{array}{ll}1 & 0 \\ 0 & s\end{array}\right) \in \mathrm{GL}_{2}^{+}(\mathbb{R})$. Then as $s \rightarrow \infty$ we have

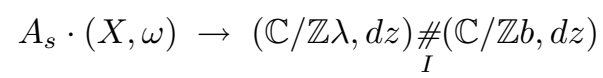

in $\Omega \overline{\mathcal{M}_{2}}$, where $I=[0, t \lambda]$.

Proof. The rank two lattices

$$
\Lambda_{1}^{s}=\mathbb{Z}(\lambda, 0) \oplus \mathbb{Z}(0, s \lambda), \quad \Lambda_{2}^{s}=\mathbb{Z}(b, 0) \oplus \mathbb{Z}(s a, s c)
$$

giving the summands of

$$
A_{s} \cdot(X, \omega)=\left(\mathbb{C} / \Lambda_{1}^{s}, d z\right) \underset{I}{\#}\left(\mathbb{C} / \Lambda_{2}^{s}, d z\right)
$$

converge geometrically to the rank one discrete groups $\mathbb{Z}(\lambda, 0)$ and $\mathbb{Z}(b, 0)$.

Proof of Theorem 4.1. Assume $\operatorname{SL}(X, \omega)$ is a lattice for the prototypical form of type $(a, b, c, e)$ and width $0<t<1$. Then by the Theorems 4.3 and 4.4 the closure of $\mathrm{GL}_{2}^{+}(\mathbb{R}) \cdot(X, \omega)$ in $\Omega \overline{\mathcal{M}}_{2}$ contains the stable Abelian differential $(Y, d x / y)$, where $Y$ is the hyperelliptic curve

$$
y^{2}=\left(x^{2}-1\right)(x+\cos (\pi \alpha))^{2}(x-\cos (\pi \beta))^{2}
$$

and $0<\alpha<\beta<1 / 2$ satisfy (4.1).

The zeros $P, Q$ of $d x / y$ lie over $x=\infty$, so their difference $P-Q$ is the divisor at infinity for (4.5). Moreover, by Theorem 1.4, $D_{\infty}$ is a limit of torsion divisors of constant order on nearby curves in $f(V)$. By Theorem 3.4, $D_{\infty}$ is itself torsion, and hence $\alpha$ and $\beta$ are rational by Corollary 3.3. 


\section{The regular decagon}

In this section we illustrate the results of the preceding section using the Teichmüller curve generated by the decagon form.

Algebraic description. Let $(X, \omega)$ denote the decagon form, given by $\omega=$ $d x / y$ on the hyperelliptic curve

$$
y^{2}=x\left(x^{5}-1\right) .
$$

By symmetry considerations, this form can be also obtained from a regular decagon $(Q, d z)$ in $\mathbb{C}$ by identifying opposite sides.

Theorem 5.1 The decagon form generates the Teichmüller

$$
f: V \rightarrow \mathcal{M}_{2}
$$

given by $f(t)=\left[X_{t}\right]$, where $X_{t}$ is the hyperelliptic curve $y^{2}=q_{t}(x)$,

$$
q_{t}(x)=x\left(-1-2 t^{5}-t^{10}+25 t^{4} x-50 t^{3} x^{2}+35 t^{2} x^{3}-10 t x^{4}+x^{5}\right),
$$

and $t$ ranges in the space $V=\left(\mathbb{P}^{1}-\left\{t: t^{10}=1\right\}\right) / D_{10}$.

Here $D_{10}$ is the dihedral group generated by $t \mapsto 1 / t$ and $t \mapsto \exp (2 \pi i / 5) t$. The curves $X_{t}$ and $X_{g t}$ are isomorphic for all $g \in D_{10}$, so $f$ is well-defined on $V$. We will also see that the 1 -forms lying over $X_{t}$ in the orbit $\mathrm{GL}_{2}^{+}(\mathbb{R}) \cdot(X, \omega)$ are proportional to $d x / y$.

The proof will be deferred to the end of the section.

Stable limits. The Teichmüller curve $f: V \rightarrow \mathcal{M}_{2}$ is also generated by the prototypical form of type $(a, b, c, e)=(0,1,1,-1)$ and $t=(2+\gamma) / 5$, where $\gamma=(1+\sqrt{5}) / 2$ is the golden mean [Mc4, Lemma 8.2]. For this prototype, we have $\lambda=\left(e+\sqrt{e^{2}+4 b c}\right) / 2=\gamma^{-1}$.

To verify Theorem 4.1 , note that as $t \rightarrow 1$, the 1 -form $\left(X_{t}, d x / y\right)$ converges to the stable Abelian differential $\left(X_{1}, d x / y\right)$ on the nodal curve

$$
y^{2}=q_{1}(x)=x(x-4)\left(1-3 x+x^{2}\right)^{2} .
$$

This curve is isomorphic to

$$
y^{2}=\left(x^{2}-1\right)\left(4 x^{2}+2 x-1\right)^{2}=\left(x^{2}-1\right)(x-A)^{2}(x-B)^{2},
$$

where $\{A, B\}=\{-\cos (\pi / 5), \cos (2 \pi / 5)\}=\{(-1 \pm \sqrt{5}) / 4\}$. Thus the corresponding ratio of sines satisfies

$$
\frac{\sin (\pi / 5)}{\sin (2 \pi / 5)}=\frac{-1+\sqrt{5}}{2}=\frac{\lambda}{b}
$$

and the width is given by

$$
t=\frac{2+\gamma}{5}=\frac{2}{5}+\frac{1}{5} \frac{b}{\lambda}
$$




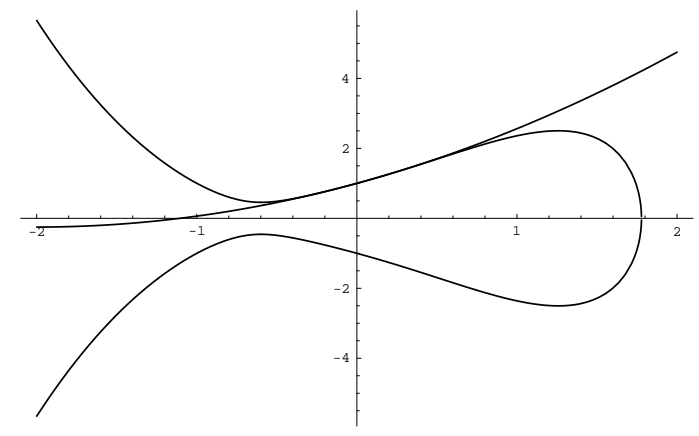

Figure 6. The divisor $D_{0}$ has order 5 .

both as predicted by (4.1). The graph of $y^{2}=\left(1-x^{2}\right)\left(4 x^{2}+2 x-1\right)$ is shown in Figure 2 of the Introduction.

Torsion divisors. Note that the zeros $P, Q \in X_{t}$ of $\omega_{t}=d x / y$ lie over $x=\infty$, so their difference $P-Q$ is the divisor at infinity. According to Theorem 1.4, this divisor is torsion whenever $X_{t}$ is irreducible. In fact, one can see explicitly that:

Theorem 5.2 For any $t$ such that $t^{5}+1 \neq 0,5 D_{\infty}$ is a principal divisor on the curve $y^{2}=q_{t}(x)$.

Proof. It is convenient to replace $q_{t}$ with its reciprocal polynomial

$$
\begin{aligned}
r_{t}(x) & =x^{6} q_{t}\left(x^{-1}\right) \\
& =1-10 t x+35 t^{2} x^{2}-50 t^{3} x^{3}+25 t^{4} x^{4}-x^{5}-2 t^{5} x^{5}-t^{10} x^{5},
\end{aligned}
$$

so that $D_{\infty}$ becomes the divisor $D_{0}$ lying over $x=0$. We then find the remarkable factorization

$$
r_{t}(x)=\left(5 t^{2} x^{2}-5 t x+1\right)^{2}-\left(t^{5}+1\right)^{2} x^{5} .
$$

This means that the conic

$$
y=f_{t}(x)=5 t^{2} x^{2}-5 t x+1
$$

intersects the curve $y^{2}=r_{t}(x)$ to order 5 over $x=0$ (see Figure 6 for the case $t=-1 / 4)$. Consequently, $F_{t}(x, y)=\left(y-f_{t}(x)\right) /\left(y+f_{t}(x)\right)$ gives a rational function with divisor $\left(F_{t}\right)=5 D_{0}$. 
Remark. The corresponding solution to Pell's equation $a_{t}(x)^{2}-b_{t}(x)^{2} q_{t}(x)=1$ satisfies

$$
b_{t}(x)=\frac{2\left(5 t^{2}-5 t x+x^{2}\right)}{\left(1+t^{5}\right)^{2}} .
$$

Regular polygons. We now proceed to the proof of Theorem 5.1.

Let $Y$ be the hyperelliptic curve $y^{2}=x^{n}-1$, and let $\xi=d x / y$. The form $(Y, \xi)$ can also be described geometrically as the quotient $(Q, d z) / \sim$, where $Q \subset \mathbb{C}$ is a pair of regular $n$-gons joined along an edge, and its other edges are identified in opposite pairs (Figure 7).

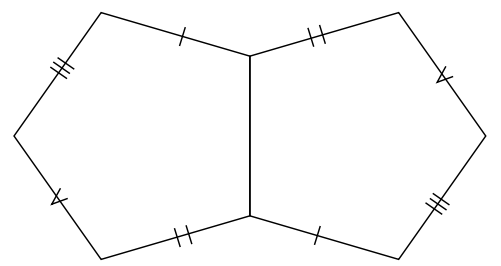

Figure 7. A polygonal model for the form $d x / y$ on the curve $y^{2}=x^{5}-1$.

It is known that $\mathrm{SL}(Y, \xi)$ is a lattice for all $n \geq 3$; in fact $\mathrm{SL}(Y, \xi)$ is a triangle group of signature $(2, n, \infty)$ when $n$ is odd, and signature $(n / 2, \infty, \infty)$ when $n$ is even $[\mathrm{V}]$.

Moreover, by [Lo] the Teichmüller curve

$$
F: V \cong \mathbb{H} / \mathrm{SL}(Y, \xi) \rightarrow \mathcal{M}_{g}
$$

can be described algebraically as follows. For $t \in \mathbb{P}^{1}$, let $Y_{t}$ be the hyperelliptic curve given by

$$
y^{2}=p_{t}(x)=\prod_{i=0}^{n}\left(x-\zeta^{i}-t \zeta^{-i}\right),
$$

where $\zeta=e^{2 \pi i / n}$. Let $G \subset \operatorname{Aut}\left(\mathbb{P}^{1}\right)$ denote the dihedral group generated by $t \mapsto 1 / t$ and $t \mapsto \zeta^{2} t$. Then the isomorphism class of $Y_{t}$ depends only on the value of $t \in \mathbb{P}^{1} / G$, and the map (5.1) is given on the quotient space

$$
V=\left(\mathbb{P}^{1}-\left\{t: t^{n}=1\right\}\right) / G
$$

simply by $F(t)=\left[Y_{t}\right] \in \mathcal{M}_{g}$. (See $[\mathrm{Me}]$ for a related construction.) The forms $\left(Y_{t}, \xi_{t}\right)=\left(Y_{t}, d x / y\right)$ all lie in the orbit $\mathrm{GL}_{2}^{+}(\mathbb{R}) \cdot(Y, \xi)$, so they also generated the Teichmüller curve $F: V \rightarrow \mathcal{M}_{g}$.

Now suppose $n=2 k$ is even. Then only even powers of $x$ occur in $p_{t}(x)$, and therefore $\rho(x, y)=(-x,-y)$ is an automorphism of $Y_{t}$. Using the change of variables $(x, y) \leftarrow\left(x^{2}, x y\right)$, the quotient $X_{t}=Y_{t} /\langle\rho\rangle$ can be expressed as the hyperelliptic curve

$$
y^{2}=q_{t}(x), \quad \text { where } q_{t}\left(x^{2}\right)=x^{2} p_{t}(x) .
$$


The form $2 d x / y$ is also $\rho$-invariant, so it descends to the form $\omega_{t}=d x / y$ on $X_{t}$. Since $\operatorname{deg} q_{t}(u)=1+k, \omega_{t}$ has a single zero (lying over $x=\infty$ ) when $k$ is even, and a pair of distinct zeros when $k$ is odd.

Note that $X_{0}$ is the hyperelliptic curve $y^{2}=q_{0}(x)=x\left(x^{k}-1\right)$. Geometrically, the form $\left(X_{0}, \omega_{0}\right)$ is obtained from a single regular $2 k$-gon $(P, d z)$ by identifying opposite sides. Thus the regular $2 k$-gon generates the Teichmüller curve

$$
f: V \rightarrow \mathcal{M}_{h}
$$

given by $f(t)=\left[X_{t}\right]=\left[y^{2}-q_{t}(x)\right]$.

Proof of Theorem 5.1. For the decagon, we simply specialize to the case $n=10$ in (5.2), and use (5.3) to obtain a formula for $q_{t}(x)$.

\section{Uniqueness}

In this section we complete the proof of our main result by showing:

Theorem 6.1 The only primitive Teichmüller curve $f: V \rightarrow \mathcal{M}_{2}$ generated by a form with simple zeros comes from the regular decagon.

Sine ratios. We begin by using the list of quadratic irrational sine ratios to show:

Theorem 6.2 Suppose the prototypical form $(X, \omega)$ of type $(a, b, c, e)$ and width $0<t<1$ generates a primitive Teichmüller curve. Let $f=\operatorname{gcd}(b, c, e)$, and write $(a, b, c, e)=(a, f B, f C, f E)$. Then $(B, C, E)$ and $t$ are equal to one of the 22 possibilities listed in Table 8.

Proof. Let $\lambda=\left(e+\sqrt{e^{2}+4 b c}\right) / 2$ as usual, and let $\mu=\left(E+\sqrt{E^{2}+4 B C}\right) /(2 B)$. Then $\mu=\lambda / b$. Since the Teichmüller curve generated by $(X, \omega)$ is primitive, $\mu$ is a quadratic irrational.

According to Theorem 4.1, there exist rationals $\alpha \in(0,1 / 2)$ and $\beta \in(0,1)$ such that

$$
\sin (\pi \alpha) / \sin (\pi \beta)=\mu \text { and } t=\beta+\alpha \mu^{-1} .
$$

Thus $\alpha$ and $\min (\beta, 1-\beta)$ must correspond to one of the 15 quadratic irrational sine ratios listed in Table 3 of $\S 2$. The values of $\alpha$ and $\beta$ determine $\mu$ and $t$ by the formulas above. They also determine the triple $(B, C, E)$, since this is the smallest positive integral vector proportional to $(1,-\mathrm{N}(\mu), \operatorname{Tr}(\mu))$.

It is thus straightforward to convert Table 3 into Table 8 , in which the values of $(\alpha, \beta)$ in the first two columns give rise to the values of $(B, C, E)$ and $t$ in the columns that follow. The cases $(\alpha, \beta)=(1 / 12,5 / 12)$ and $(1 / 10,3 / 10)$ in Table 3 are excluded, because they give $c / b=-N(\mu)<0$, contrary to the condition $b, c>0$ in the definition of a prototype. 


\begin{tabular}{|c|c|c|c|}
\hline$\alpha$ & $\beta$ & $(B, C, E)$ & $t$ \\
\hline \hline $1 / 10$ & $1 / 2$ & $(4,1,-2)$ & $(6+\sqrt{5}) / 10$ \\
\hline $1 / 12$ & $1 / 4$ & $(2,1,-2)$ & $(4+\sqrt{3}) / 12$ \\
\hline $1 / 12$ & $3 / 4$ & $(2,1,-2)$ & $(10+\sqrt{3}) / 12$ \\
\hline $1 / 8$ & $3 / 8$ & $(1,1,-2)$ & $(4+\sqrt{2}) / 8$ \\
\hline $1 / 8$ & $5 / 8$ & $(1,1,-2)$ & $(6+\sqrt{2}) / 8$ \\
\hline $1 / 6$ & $1 / 3$ & $(3,1,0)$ & $(2+\sqrt{3}) / 6$ \\
\hline $1 / 6$ & $2 / 3$ & $(3,1,0)$ & $(4+\sqrt{3}) / 6$ \\
\hline $1 / 6$ & $3 / 10$ & $(1,1,-1)$ & $(23+5 \sqrt{5}) / 60$ \\
\hline $1 / 6$ & $7 / 10$ & $(1,1,-1)$ & $(47+5 \sqrt{5}) / 60$ \\
\hline $1 / 10$ & $1 / 6$ & $(1,1,-1)$ & $(13+3 \sqrt{5}) / 60$ \\
\hline $1 / 10$ & $5 / 6$ & $(1,1,-1)$ & $(53+3 \sqrt{5}) / 60$ \\
\hline $1 / 5$ & $2 / 5$ & $(1,1,-1)$ & $(5+\sqrt{5}) / 10$ \\
\hline $1 / 5$ & $3 / 5$ & $(1,1,-1)$ & $(7+\sqrt{5}) / 10$ \\
\hline $1 / 6$ & $1 / 4$ & $(2,1,0)$ & $(3+2 \sqrt{2}) / 12$ \\
\hline $1 / 6$ & $3 / 4$ & $(2,1,0)$ & $(9+2 \sqrt{2}) / 12$ \\
\hline $1 / 4$ & $1 / 2$ & $(2,1,0)$ & $(2+\sqrt{2}) / 4$ \\
\hline $1 / 4$ & $5 / 12$ & $(1,2,-2)$ & $(13+3 \sqrt{3}) / 24$ \\
\hline $1 / 4$ & $7 / 12$ & $(1,2,-2)$ & $(17+3 \sqrt{3}) / 24$ \\
\hline $3 / 10$ & $1 / 2$ & $(4,1,2)$ & $(2+3 \sqrt{5}) / 10$ \\
\hline $1 / 4$ & $1 / 3$ & $(3,2,0)$ & $(8+3 \sqrt{6}) / 24$ \\
\hline $1 / 4$ & $2 / 3$ & $(3,2,0)$ & $(16+3 \sqrt{6}) / 24$ \\
\hline $1 / 3$ & $1 / 2$ & $(4,3,0)$ & $(9+4 \sqrt{3}) / 18$ \\
\hline
\end{tabular}

Table 8. The 22 candidates. 
Ratios of moduli. To complete the proof, we will use the following rationality constraint.

Theorem 6.3 If the prototypical form $(X, \omega)$ of type $(a, b, c, e)$ and width $t$ generates a Teichmüller curve, then the ratio

$$
\frac{(1-t)(b c / g+(n+1) \lambda)}{t \lambda-n g}
$$

is rational, where $\lambda=\left(e+\sqrt{e^{2}+4 b c}\right) / 2, g=\operatorname{gcd}(a, b)$ and $n=\lfloor t \lambda / g\rfloor$.

(Here $\lfloor x\rfloor$ denotes the greatest integer less than or equal to $x$.)

Proof. Consider the foliation $\mathcal{F}$ of $(X,|\omega|)$ by vertical geodesics, corresponding to vertical lines in the polygon $P \subset \mathbb{C}$ in Figure 4 . The first return map of $\mathcal{F}$ to the transversal $L=[0, b]$, forming the base of the upper parallelogram in $P$, is given by $f(x)=x+a \bmod b$. Since $f$ is periodic, all the leaves of $\mathcal{F}$ are closed.

More precisely, the leaves of $\mathcal{F}$ disjoint from $L$ sweep out a cylinder $C_{1}$ of height $h_{1}=(1-t) \lambda$ and circumference $c_{1}=\lambda$. The leaves that do meet $L$ cross it $b / g$ times, since this is the period of $f$. These leaves sweep out a pair of cylinders $C_{2}$ and $C_{3}$, distinguished by their intersection numbers with $I=[0, t \lambda] \subset L$ : we have $C_{2} \cdot I=n$ and $C_{3} \cdot I=n+1$. Since $C_{i} \cdot I$ also counts the number of times that $C_{i}$ passes through the $\lambda \times \lambda$ square inside of $P$, these cylinders have circumferences $c_{2}=b c / g+n \lambda$ and $c_{3}=b c / g+(n+1) \lambda$. Similarly one can check that their heights are given by $h_{2}=(n+1) g-t \lambda$ and $h_{3}=t \lambda-n g$.

Now if $\operatorname{SL}(X, \omega)$ is a lattice, then the moduli $m_{i}=h_{i} / c_{i}$ of the cylinders $C_{i}$ have rational ratios (see e.g. [V, 2.4], [Mc1, Lemma 9.7]). In particular, the ratio $m_{1} / m_{3}$ given by (6.1) is rational.

Example. For the prototypical form $(X, \omega)$ of type $(a, b, c, e)=(0,1,1,-1)$ and width $t=(2+\gamma) / 5$ (generating the regular decagon), the moduli of the 3 cylinders of the vertical foliation satisfy $\left[m_{1}: m_{2}: m_{3}\right]=[1: 2: 1]$. We have $n=0, g=1$, and the quantity (6.1) is simply 1 .

Proof of Theorem 6.1. Let $f: V \rightarrow \mathcal{M}_{2}$ be a primitive Teichmüller curve generated by a holomorphic 1 -form $(X, \omega)$ with simple zeros. By Theorem 4.2 we may assume $(X, \omega)$ is a prototypical form, of type $(a, b, c, e)$ and width $0<t<1$. Let $f=\operatorname{gcd}(b, c, e)$ and write $(a, b, c, e)=(a, f B, f C, f E)$; then $(B, C, E)$ and $t$ agree with one of the 22 possibilities listed in Table 8 .

By the definition of a prototype, we have $\operatorname{gcd}(a, b, c, e)=\operatorname{gcd}(a, f)=1$. Therefore

$$
g=\operatorname{gcd}(a, b)=\operatorname{gcd}(a, f B)=\operatorname{gcd}(a, B)
$$

is a divisor of $B$. So for each value of $(B, C, E, t)$, there are only finitely many possibilities for $g$.

For concreteness, suppose the candidate prototype satisfies $(B, C, E)=$ $(1,1,-1)$ and $t=(5+\sqrt{5}) / 10$. Then $\mu=\lambda / b=(-1+\sqrt{5}) / 2$, and $g=1$. 
Substituting $(b, c)=(f B, f C)$ and $\lambda=\mu f B$ into (6.1), Theorem 6.3 implies the ratio

$$
\frac{f\left(3 f-f^{2}-5 n+8 f n-5 n^{2}+\sqrt{5}\left(f^{2}-2 f n+3 n^{2}-f+3 n\right)\right)}{2\left(f^{2}-5 n^{2}\right)}
$$

is rational, where

$$
n=\lfloor t f B \mu / g\rfloor=\lfloor f / \sqrt{5}\rfloor .
$$

Equivalently, we have

$$
p(f, n)=f^{2}-2 f n+3 n^{2}-f+3 n=0 .
$$

It is straightforward to verify that the only positive integral solution to $(6.2)$ (with $n=\lfloor f / \sqrt{5}\rfloor$ ) is $f=1$. Indeed, for large values of $f$ we have $n \approx f / \sqrt{5}$, and thus $p(f, n)$ grows like $f^{2}$, leaving only finitely many cases to check. (A plot of $p(f,\lfloor f / \sqrt{5}\rfloor)$ is shown in Figure 9$)$.

The case $f=1$ gives $(a, b, c, e)=(0,1,1,-1)$, so it corresponds to the regular decagon.

A similar calculation shows there are no Teichmüller curves at all coming from the remaining candidates in Table 8. For example, suppose $(B, C, E)=$ $(2,1,-2), t=(4+\sqrt{3}) / 12$ and $g=2$. Then $\mu=(1+\sqrt{3}) / 2$, and we find

$$
p(f, n)=23 f^{2}-48 f n+216 n^{2}-24 f+216 n=0,
$$

where $n=\lfloor(-1+3 \sqrt{3}) f / 24\rfloor$. For $f=1,2,3,4,5, \ldots$ we have $p(f, n)=$ $-1,44,135,272,455, \ldots$, and quadratic growth insures $p(f, n)>0$ for all $f>5$.

The remaining cases are similar.

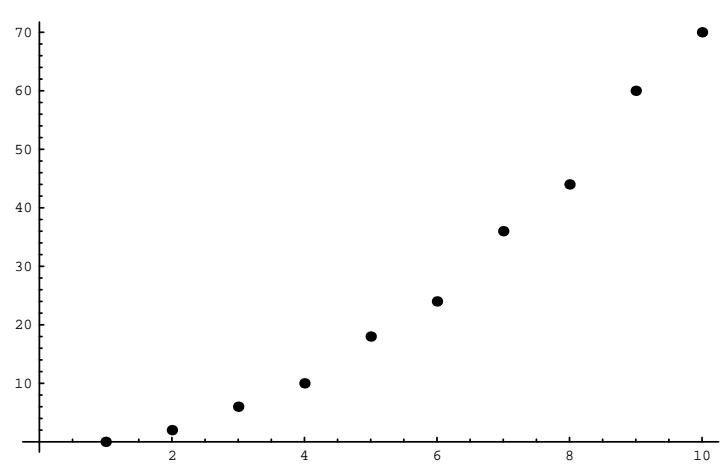

Figure 9. Plot of $p(f,\lfloor f / \sqrt{5}\rfloor)$. 


\section{References}

[Ab] N. H. Abel. Über die Integration der Differential-Formel $\rho d x / \sqrt{R}$, wenn $R$ und $\rho$ ganze Funktionen sind. J. reine angew. Math. 1(1826), 185-221.

[BC] E. Bombieri and P. B. Cohen. Siegel's lemma, Padé approximations and Jacobians. Ann. Scuola Norm. Sup. Pisa Cl. Sci. (4) 25(1997), $155-178$.

[BGL] J. Boxall, D. Grant, and F. Leprévost. 5-torsion points on curves of genus 2. J. London Math. Soc. 64(2001), 29-43.

[Ca] K. Calta. Veech surfaces and complete periodicity in genus 2. J. Amer. Math. Soc. 17(2004), 871-908.

[EMS] A. Eskin, H. Masur, and M. Schmoll. Billiards in rectangles with barriers. Duke Math. J. 118(2003), 427-463.

[GJ] E. Gutkin and C. Judge. Affine mappings of translation surfaces: geometry and arithmetic. Duke Math. J. 103(2000), 191-213.

[HM] J. Harris and I. Morrison. Moduli of Curves. Springer-Verlag, 1998.

[HMP] Y. Hellegouarch, D. L. McQuillan, and R. Paysant-Le Roux. Unités de certains sous-anneaux des corps de fonctions algébriques. Acta Arith. 48(1987), 9-47.

[HL] P. Hubert and S. Lelièvre. Square-tiled surfaces in $\mathcal{H}(2)$. Preprint, 2004.

[HS] P. Hubert and T. A. Schmidt. Infinitely generated Veech groups. Duke Math. J. 123(2004), 49-69.

[Lo] P. Lochak. On arithmetic curves in the moduli space of curves. Preprint, 2003.

[Man] H. B. Mann. On linear relations between roots of unity. Mathematika 12(1965), 107-117.

[Mc1] C. McMullen. Billiards and Teichmüller curves on Hilbert modular surfaces. J. Amer. Math. Soc. 16(2003), 857-885.

[Mc2] C. McMullen. Teichmüller geodesics of infinite complexity. Acta Math. 191(2003), 191-223.

[Mc3] C. McMullen. Teichmüller curves in genus two: Discriminant and spin. Math. Ann. 333(2005), 87-130.

[Mc4] C. McMullen. Teichmüller curves in genus two: The decagon and beyond. J. reine angew. Math. 582(2005), 173-200. 
[Me] J.-F. Mestre. Familles de courbes hyperelliptiques à multiplications réelles. In Arithmetic Algebraic Geometry (Texel, 1989), volume 89 of Progr. Math., pages 193-208. Birkhaüser Boston, 1991.

[Mo] M. Möller. Periodic points on Veech surfaces and the Mordell-Weil group over a Teichmüller curve. Preprint, 4/2005.

[Ri] T. J. Rivlin. The Chebyshev Polynomials. Wiley, 1974.

[V] W. Veech. Teichmüller curves in moduli space, Eisenstein series and an application to triangular billiards. Invent. math. 97(1989), 553-583.

[Yu J. Yu. On arithmetic of hyperelliptic curves. In N. Mok, editor, Aspects of Mathematics: Algebra, Geometry and Several Complex Variables, pages 395-415. University of Hong Kong, 2001.

\author{
Mathematics Department \\ HARVARD UNIVERSITY \\ 1 OXFORD ST \\ CAmbridge, MA 02138-2901
}

